\title{
BMJ Open Exploring the operationalisation and implementation of outreach in community settings with hard-to-reach and hidden populations: protocol for a scoping review
}

\author{
Janina Krabbe (D) , ${ }^{1}$ Sunny Jiao (D) , ${ }^{2}$ Adrian Guta, ${ }^{3}$ Allie Slemon (D) , \\ Aman Ahluwalia Cameron (iD, ${ }^{3}$ Vicky Bungay (iD ${ }^{2}$
}

To cite: Krabbe J, Jiao S, Guta A, et al. Exploring the operationalisation and implementation of outreach in community settings with hard-to-reach and hidden populations: protocol for a scoping review. BMJ Open 2021;11:e039451. doi:10.1136/ bmjopen-2020-039451

- Prepublication history and additional material for this paper are available online. To view these files, please visit the journal online (http://dx.doi. org/10.1136/bmjopen-2020039451).

Received 16 April 2020 Revised 20 0ctober 2020 Accepted 27 January 2021

Check for updates

(c) Author(s) (or their employer(s)) 2021. Re-use permitted under CC BY-NC. No commercial re-use. See rights and permissions. Published by BMJ.

1 Interdisciplinary Studies, University of British Columbia, Vancouver, British Columbia, Canada

${ }^{2}$ School of Nursing, University of British Columbia, Vancouver, British Columbia, Canada

${ }^{3}$ School of Social Work,

University of Windsor, Windsor, Ontario, Canada

Correspondence to

Dr Vicky Bungay;

vicky.bungay@ubc.ca

\section{ABSTRACT}

Introduction Outreach is regularly identified as an effective strategy to engage underserved, hard-to-reach and hidden populations with essential life-sustaining health services. Despite the increasing expansion of outreach programmes, particularly in HIV prevention and health promotion with youth, sex workers, people living with mental health and substance use challenges, and those affected by homelessness, there has been limited synthesis of the evidence concerning the core components of outreach programming or indicators of its successful implementation. Without this understanding, current outreach programmes may be limited in achieving the desired aims. The aim of this scoping review is to explore how outreach has been operationalised and implemented in various community settings with people underserved in current healthcare contexts. Understanding the state of knowledge pertaining to outreach as programming and as practice involving the engagement of people considered hard-to-reach will enable the identification of promising trends and limitations in the field.

Methods and analysis This scoping review follows the Arksey and 0'Malley's framework. CINAHL, MEDLINE, PsycINF0 and PubMed databases will be searched for peer-reviewed references focused on outreach with hardto-reach and hidden groups from 1 January 2008 to 30 April 2020. Guided by explicit inclusion and exclusion criteria, three reviewers will independently assess references in two successive stages. Titles and abstracts will be reviewed followed by full-text assessment of papers meeting the review criteria. A descriptive overview, tabular and/or graphical summaries and a thematic analysis will be carried out on extracted data.

Ethics and dissemination Ethics approval was not required as the only data source was peer-reviewed documents. Outreach knowledge users who are members of the project team will participate in all aspects of study design, implementation and result dissemination strategies.

\section{INTRODUCTION}

Outreach is regularly identified as a recommended strategy to provide support, address

\section{Strengths and limitations of this study}

- This is the first scoping review to systematically examine the definitions, programme components and indicators of successful implementation of outreach activities, allowing the mapping of this area of research through the summarisation of volume, nature and characteristics of the available work and the identification of knowledge gaps.

- Although a comprehensive search strategy was developed in consultation with a health science librarian, enabling a nuanced and rigorous approach to literature sources, the populations, concept (outreach) and domain of interest (health and social inequities) were indexed using a variety of terms, which poses challenges to ensuring the breadth and depth of the search.

- Quality appraisal tools are integrated into data extraction to promote description of both the topics of interest and the quality of research undertaken in the topic area.

- This review is conducted by a multidisciplinary team including knowledge user experts, who can support the use of study findings in real-world applications across policy and health and social services.

- The methodology is capable of encompassing findings from a wider range of study designs and methods in comparison with a systematic review, but is only capable of generating a narrative or descriptive account of the available research and does not account for the relative weight of evidence.

barriers to accessible healthcare and foster health-promoting practices among groups commonly described as hard-to-reach and hidden populations. ${ }^{12}$ Although definitions of this term vary across the literature, hard-toreach and hidden are defined in this study as populations that have uncertain parameters such as the size and geographical distribution of the population, experience intersecting 
forms of stigma and discrimination, may conceal membership in a particular social group, and potentially distrust researchers or healthcare providers who they believe have contributed to stereotyping and unjust social policies and practices. ${ }^{3-5}$ People situated within hard-to-reach and hidden populations regularly experience significant health inequities, defined as avoidable differences in health status and the determinants of health between population groups. ${ }^{6}$ Women simultaneously affected by poverty and violence, for instance, experience multiple chronic illnesses, unstable housing and shorter life expectancy than other women in society. ${ }^{7}$ People engaged in illegal drug use and those with mental health issues, homelessness and criminal justice involvement are exponentially more vulnerable to HIV and accidental overdose, situations exacerbated by structural inequities in resources necessary for health. ${ }^{5}$

The health inequities experienced by hard-to-reach and hidden populations are further reinforced through structural inequities (ie, poverty, discrimination) and exacerbated by barriers to appropriate, timely healthcare..$^{8-10}$ Such barriers include knowledge gaps concerning available services, competing needs to secure food and shelter, and the unavailability (eg, timing, location) of services necessary to meet their needs. ${ }^{11-14}$ People considered hard-to-reach or hidden also regularly experience discriminatory interactions within healthcare encounters that result in unmet health needs and future reluctance to engage with these services. ${ }^{911} 1516$ Consequently, hardto-reach and hidden populations are chronically underserved in current health services, thereby contributing to the urgent need to redress health inequities among such groups. ${ }^{1718}$

Presently, outreach is increasingly employed by nurses, social workers and community health workers to enhance health service delivery with hard-to-reach and hidden populations. ${ }^{12}$ Outreach programmes are also expanding throughout the world among varied hardto-reach and hidden populations, including migrant populations, 'at risk' youth, sex workers, women experiencing violence, people who use illegal drugs and individuals who are homeless or experiencing mental health challenges. $^{1219}$ These outreach programmes commonly occur within interdisciplinary, team-based, care provision contexts associated with primary care and/or community health clinics. ${ }^{19}$

While the importance of outreach to enhance healthcare delivery with hard-to-reach and hidden populations appears to be consistent across various practice contexts, it is unclear if the meaning of outreach is programmespecific or discipline-specific or if outreach varies based on the different populations served (eg, migrant populations vs homeless women). It is also unclear how outreach is implemented or operationalised into its core elements and subsequently evaluated for effectiveness. The consequences of oversights in examining the theoretical tenets and core elements of outreach contribute to significant challenges in developing and sustaining effective evidence-informed outreach programming. Programme evaluation to assess the effectiveness of outreach to meet programmatic aims in health service delivery may be seriously undermined. Moreover, without a shared understanding of the concept and core components, it is almost impossible to identify essential research priorities in advancing the empirical evidence to develop, implement and evaluate outreach programming. To our knowledge, it is not known to what extent outreach has been systematically defined, operationalised or implemented. Bringing this evidence together in a systematic scoping review has the potential to identify intersecting, disciplinary and population-specific dimensions of outreach that have been defined, implemented and empirically explored.

\section{OBJECTIVE}

This scoping review will systematically explore how outreach has been operationalised and implemented in diverse community settings with varied hard-to-reach and hidden populations and enable identification of promising trends and limitations in current research.

\section{METHODS AND ANALYSIS}

The scoping review methodology will employ the systematic framework developed by Arksey and O'Malley, ${ }^{20}$ advancements from Levac and colleagues, ${ }^{21}$ and best practices in reporting and conducting systematic reviews including the Preferred Reporting Items for Systematic Review and Meta-Analysis Protocols. ${ }^{22}$ Consultation with knowledge users is an integral aspect of the usefulness of a scoping review. Given the interdisciplinarity of outreach work, encompassing nursing, social work and public health, our team composition reflects this interdisciplinarity inclusive of members from each of these applied disciplines with research and practice expertise. An iterative approach will be maintained during the screening of studies, data extraction and consultations with knowledge user experts, all of which will become more refined throughout the review. The authors will use NVivo for Teams V. $12^{23}$ to support the screening of studies, data extraction and analysis.

\section{Stage 1: determine review aims}

To understand how outreach is operationalised and implemented, the research questions were developed to support a wide-ranging description of the populations (ie, those considered hard-to-reach, hidden and underserved in current health services), the core activities being investigated (ie, outreach) and the context in which outreach services occur (ie, the health and social issues that precipitate the need for outreach services). Therefore, the questions addressed in the review are the following:

- How has outreach been defined within health-oriented programmes for people situated in hard-to-reach and hidden populations? 
- How has outreach been operationalised as healthoriented programmes for people situated in hard-toreach or hidden populations?

- How has outreach been implemented as health-oriented programmes for people situated in hard-to-reach or hidden populations?

- What outcomes from the implementation of outreach have been empirically measured or tested?

Answering these questions will help to identify limitations and strengths of the current state of knowledge and to generate recommendations for future research priorities.

\section{Stage 2: identify relevant literature}

The uncertain consistency in how outreach is defined, operationalised, implemented and evaluated across various groups and settings makes our proposed topic inherently complex and poses significant challenges to keyword selection. For instance, outreach as practice is carried out across diverse sectors of health and social services and the term may be used to represent different actions or goals depending on the disciplinary focus. Likewise, the language used to describe target populations that have historically been served by outreach has undergone significant shifts, for instance from 'addicts' or 'juvenile delinquents' to health-oriented and person-oriented language such as 'people with problematic substance use' or 'at-risk youth'. The language of health systems has also begun to shift from terms such as 'non-compliant' or 'difficult patients' to 'underserved', 'vulnerable' or 'marginalised populations and groups'.

Consequently, we developed a comprehensive search strategy that promotes consideration of this complexity while simultaneously enabling a systematic review of the existing evidence. Initially, we consulted with eight knowledge users who were practice experts working in outreach in various healthcare organisations. Accordingly, we gained insights into existing outreach programmes and practices and began to understand the assorted contexts in which outreach occurs. We also had extensive consultations with a health sciences research librarian at the University of British Columbia. From these consultations, we identified three intended domains for our search strategy: (1) the concept domain of outreach as a programme or practice; (2) the population domain (hard-to-reach and hidden groups); and (3) the context domain (health and social issues, for instance stigma, barriers to healthcare, homelessness and problematic substance use that precipitate the need for outreach services).

Four electronic databases, CINAHL, MEDLINE, PubMed and PsycINFO, were identified to optimise our ability to capture the breadth and depth of published scholarship pertinent to outreach programmes and practices. These databases are critical to the health and social science disciplines, thereby permitting exploration in an interdisciplinary context. To develop the appropriate keywords for the searches, initial search terms were first inputted into each database to determine corresponding indexed terms. For example, PubMed uses Medical Subject Headings (MeSH) terms, while CINAHL, MEDLINE and PsycINFO use subject terms. Each resulting indexed term (MeSH or subject term) was assessed by two authors ( $\mathrm{SJ}$ and $\mathrm{JK}$ ) to determine its utility in addressing our research questions. Indexed terms' scope notes-the database-specific definition it ascribed to the meaning and interpretation of that term-guided the assessment. Any assessment discrepancies between the authors or questions about term inclusion were brought to the larger team, who made decisions based on their expertise in the content area and insights from the previous consultations with knowledge users. This process resulted in search strategies for each of the four databases (see online supplemental 1 for the step-by-step process details of formulating a search strategy and online supplemental 2 for the precise search strategy for one of the databases, CINAHL). Boolean operators ('and', 'or', 'not') will be used to combine and refine search terms.

\section{Stage 3: study selection}

Study selection will include a two-stage process that incorporates predetermined assessment strategies and inclusion criteria developed collaboratively within the study team inclusive of knowledge users. In stage 1, the screening process will be piloted with 20 citations each from initial PubMed and CINAHL searches to test the criteria and reviewer agreement using both subject and MeSH terms. Then all four databases will be searched, and each title/abstract from the search results will be reviewed independently by two team members using eligibility criteria (SJ and JK). The reviewers' agreement will be assessed and a third reviewer (VB) will be consulted to reach consensus. A citation will be eligible for full-text screening if the title/abstract:

- Refers to outreach as a programme or practice.

- Is located in a community-based or primary healthcare setting including healthcare or social service delivery.

- Is about developing, implementing or evaluating outreach with hard-to-reach or hidden populations or in the context of health or social issues that precipitate the need for outreach, including barriers to healthcare, health and social inequities, stigma, and discrimination.

- Is geographically situated in countries with developed economies.

- Is a peer-reviewed article published in English between 1 January 2008 and 30 April 2020.

Only peer-reviewed articles, including empirical and discussion papers, will be considered. Grey literature will be excluded as the focus of this review is on the core components of outreach identified across the research literature, and empirical analysis of the implementation and evaluation of outreach. The publication period of approximately the last 10 years was decided on through various considerations. We wanted to include the most recent references, within the limits of the publication 
process, to ensure relevancy. Lastly, the approximate 10-year window allows for the inclusion of a sufficient number of references for a robust analysis.

The second phase will include obtaining the full text of references meeting the inclusion criteria. Those included will be screened, sorted by study design and assessed using the corresponding Joanna Briggs Institute Critical Appraisal Tool (online supplemental 3). ${ }^{24}$ With curated checklists specific to different study designs, the Joanna Briggs Institute Critical Appraisal Tools are an effective means of determining the quality of references across multiple reviewers. Two team members $(\mathrm{SJ}$ and $\mathrm{JK}$ ) will independently appraise reference quality and screen them for a definition of outreach. The reviewers' agreement will be assessed and a third reviewer (VB) will be consulted to reach consensus. This process will enable quality references with a definition of outreach to be included for data extraction.

\section{Stage 4: data extraction}

The research team will use NVivo for Teams V.12 ${ }^{23}$ to organise references and delete duplicates. As recommended by Arksey and O'Malley, we will record key study details to organise the charting of the materials generated in the search in order to distil the most pertinent information to answer our research questions (table 1 ) ${ }^{20}$ The authors (SJ, JK and AS) will pilot the data extraction and charting form with five references. The authors will then meet as a full team to discuss and adapt the form as needed.

\section{Stage 5: collating, summarising and reporting the results}

A descriptive overview of the eligible articles in graphical and chart form will be provided detailing study designs, geographical locations, and core elements of outreach programmes. ${ }^{20}$ These studies and other literature sources will also be summarised by broader categories of varied population groups and health and social issues. Narrative summaries will accompany all graphs and charts to correlate these findings to the research questions. This initial analytic step will provide perspectives on the most common elements of outreach programmes and core operational definitions, and highlight similarities and differences that are population-specific. This will assist in identifying extant gaps in knowledge and practice that can be used to inform future research priorities.

Because we are concerned with understanding how outreach has been defined, implemented, and evaluated in healthcare contexts (inclusive of social services) for hard-to-reach and hidden populations, a thematic analysis will also be carried out on the included papers. ${ }^{25}$ This will permit us to identify core elements of outreach programmes and practices as well as how definitions of outreach are operationalised in implementation and evaluation in the context of hard-to-reach and hidden populations. VB and AG will lead the thematic analysis as they have prior expertise in this area. Data will be imported into NVivo for Teams V.12, ${ }^{23}$ which permits a multi-user approach. As is appropriate in thematic analysis, a coding
Table 1 Data extraction and charting

\begin{tabular}{|c|c|}
\hline Domain/subdomain & Description \\
\hline \multicolumn{2}{|l|}{$\begin{array}{l}\text { 1. General document } \\
\text { details }\end{array}$} \\
\hline 1.1 Author(s) & Name(s) \\
\hline 1.2 Author(s)' discipline(s) & $\begin{array}{l}\text { Author(s)' discipline(s) or } \\
\text { professional credentials }\end{array}$ \\
\hline 1.3 Reference type & $\begin{array}{l}\text { Empirical study, review, non- } \\
\text { empirical }\end{array}$ \\
\hline 1.4 Publication location & Country of publication \\
\hline 1.5 Year of publication & Publication year \\
\hline 1.6 Research location & Country of research \\
\hline
\end{tabular}

2A. Empirical research study details

2.1 Objectives

What was/were the stated research objective(s) or research question(s)?

\subsection{Study design}

What was the study design?

2.3 Outreach service providers

What group of service providers was providing outreach (e.g., nurses, peers, etc.)?

2.4 Outreach recipients

Who are the recipients of outreach? Can they be classified as hard-to-reach and hidden?

2.5 Setting
$\begin{aligned} & \text { 2.6 Methodology and } \\ & \text { methods }\end{aligned}$

In what programme is outreach embedded (e.g., community healthcare, clinic, etc.)? methods What methodology and methods guided the implementation of the study?

2.7 Study population What were the eligibility/ inclusion criteria? What was the primary population of focus?

2.8 Outreach definition

What was the definition of outreach used? Was it implicitly or explicitly defined?

2.9 Results What were the main findings?

2B. Other reference types details

\begin{tabular}{|c|c|}
\hline $\begin{array}{l}2.1 \text { Non-empirical type of } \\
\text { article }\end{array}$ & $\begin{array}{l}\text { Discussion, commentary, non- } \\
\text { empirical report }\end{array}$ \\
\hline 2.2 Health or social issue & $\begin{array}{l}\text { What is the health or social } \\
\text { issue precipitating the need for } \\
\text { outreach services? }\end{array}$ \\
\hline 2.3 Objectives & $\begin{array}{l}\text { What was/were the stated } \\
\text { objective(s) of the reference? }\end{array}$ \\
\hline $\begin{array}{l}2.4 \text { Outreach service } \\
\text { providers }\end{array}$ & $\begin{array}{l}\text { What group of service providers } \\
\text { was providing outreach (e.g., } \\
\text { nurses, peers, etc.)? }\end{array}$ \\
\hline 2.5 Outreach recipients & $\begin{array}{l}\text { Who are the recipients of } \\
\text { outreach? Can they be classified } \\
\text { as hard-to-reach and hidden? }\end{array}$ \\
\hline
\end{tabular}

Continued 


\begin{tabular}{ll}
\hline Table 1 Continued & \\
\hline Domain/subdomain & Description \\
\hline 2.6 Setting & $\begin{array}{l}\text { In what programme is outreach } \\
\text { embedded (e.g., community } \\
\text { healthcare, clinic, etc.)? }\end{array}$ \\
\hline $\begin{array}{l}\text { What were the eligibility/ } \\
\text { inclusion criteria (if applicable)? } \\
\text { What was the primary } \\
\text { population of focus? }\end{array}$ \\
$\begin{array}{l}\text { What was the definition of } \\
\text { outreach used? Was it implicitly } \\
\text { or explicitly defined? }\end{array}$ \\
$\begin{array}{l}\text { What were the main messages } \\
\text { or conclusions? }\end{array}$ \\
\hline
\end{tabular}

framework will be developed a priori and then applied by VB and AG independently. Results of the thematic analysis will be summarised, and if relevant, numerical summaries may also be used to provide additional context for the themes (e.g., number of elements of outreach, number of populations served, etc.).

\section{Stage 6: consultation with knowledge users}

In keeping with the iterative nature of the scoping review approach, consultation with knowledge users will occur throughout the entire project and culminate in finalising the results and planning for dissemination. This scoping review will produce evidence about outreach that can be used by health and social service providers, researchers and policy makers on how best to provide outreach with hard-to-reach and hidden populations. Nursing, public health and social work knowledge users with expertise in outreach will be involved in providing essential insights into the relevance and meaning of search terms and the implications of the review. ${ }^{21}$ Knowledge users will participate in the analysis and in the dissemination of study results for policy and programme knowledge users.

\section{PATIENT AND PUBLIC INVOLVEMENT STATEMENT}

Researchers and knowledge users who are members of the clinical practice community are included in this scoping review, which is a product of ongoing collaborations. Knowledge users and researchers collaboratively identified the purpose and need for this scoping review while working to develop training resources for outreach staff. Knowledge users have participated in search term selection and will further contribute to the review through the analysis of results and the appropriate dissemination of review findings. Because the focus of the scoping review is on programming versus recipients of outreach, patients were not invited to contribute to the writing or editing of this document for readability or accuracy.

\section{ETHICS AND DISSEMINATION}

The scoping review protocol does not require ethics approval in accordance with the guidelines set forth by our institution for research with human participants. All data sources are peer-reviewed materials.

With the increasing focus on promoting health equity, the need for outreach services responsive to the healthcare needs of hard-to-reach and hidden populations is increasing. Understanding the theory, operational tenets and evaluation indicators may catalyse more effective outreach programming. To date, we are not aware of another scoping review that has explored the core elements of outreach programmes in community settings for hard-to reach and hidden populations.

The need for this review was identified through collaborations between researchers and knowledge users. Therefore, the dissemination strategy will include traditional academic avenues, such as open-access, peerreviewed journals, as well as health service-oriented venues, including a community report-back event. The research team's network of outreach knowledge users will be employed to determine the most appropriate dissemination strategies to local service organisations.

\section{Twitter Vicky Bungay @vickybungay}

Acknowledgements We would like to acknowledge Katherine Miller, Nursing Librarian (University of British Columbia), for her invaluable time and expertise given to inform the search strategies of this scoping review.

Contributors All authors participated fully in the design of the work, drafting and revisions of the manuscript, and are accountable for all aspects of the work. Each author approved the final version. JK, SJ and AS specifically coordinated project meetings and met with librarians to review the search strategy, prepared drafts of the search strategy and collated feedback from the project team. VB oversaw the entire project team, prepared drafts of the manuscript and finalised contributions of the team to prepare the manuscript for publication. $A G$ and $A A C$ as expert clinicians and researchers, contributed to determining search terms, data analysis approaches and identification of the role of knowledge users in dissemination and analysis.

Funding This work was supported by the Social Sciences and Humanities Research Council (SSHRC) (Partnership Development Grant: 890-2016-0016) and by VB's Canada Research Chair Tier II in Gender, Equity and Community Engagement. VB is also supported by the Michael Smith Foundation for Health Research Scholar Program.

Competing interests None declared.

Patient consent for publication Not required.

Provenance and peer review Not commissioned; externally peer reviewed.

Supplemental material This content has been supplied by the author(s). It has not been vetted by BMJ Publishing Group Limited (BMJ) and may not have been peer-reviewed. Any opinions or recommendations discussed are solely those of the author(s) and are not endorsed by BMJ. BMJ disclaims all liability and responsibility arising from any reliance placed on the content. Where the content includes any translated material, BMJ does not warrant the accuracy and reliability of the translations (including but not limited to local regulations, clinical guidelines, terminology, drug names and drug dosages), and is not responsible for any error and/or omissions arising from translation and adaptation or otherwise.

Open access This is an open access article distributed in accordance with the Creative Commons Attribution Non Commercial (CC BY-NC 4.0) license, which permits others to distribute, remix, adapt, build upon this work noncommercially, and license their derivative works on different terms, provided the original work is properly cited, appropriate credit is given, any changes made indicated, and the use is non-commercial. See: http://creativecommons.org/ licenses/by-nc/4.0/. 


\section{ORCID iDs}

Janina Krabbe http://orcid.org/0000-0002-2959-774X

Sunny Jiao http://orcid.org/0000-0002-4120-4101

Allie Slemon http://orcid.org/0000-0003-4554-7109

Aman Ahluwalia Cameron http://orcid.org/0000-0002-4805-1806

Vicky Bungay http://orcid.org/0000-0001-6440-7182

\section{REFERENCES}

1 Andersson $\mathrm{B}$. Finding ways to the hard to reach-considerations on the content and concept of outreach work. European Journal of Social Work 2013;16:171-86.

2 Lhussier M, Carr SM, Forster N. A realist synthesis of the evidence on outreach programmes for health improvement of traveller communities. J Public Health 2016;38:e125-32.

3 Benoit C, Jansson M, Millar A, et al. Community-academic research on hard-to-reach populations: benefits and challenges. Qual Health Res 2005;15:263-82.

4 Watters JK, Biernacki P. Targeted sampling: options for the study of hidden populations. Soc Probl 1989;36:416-30.

5 Bungay V, Oliffe J, Atchison C. Addressing underrepresentation in sex work research: reflections on designing a purposeful sampling strategy. Qual Health Res 2016;26:1-13.

6 Browne AJ, Varcoe C, Ford-Gilboe M, et al. Disruption as opportunity: impacts of an organizational health equity intervention in primary care clinics. Int J Equity Health 2018;17:154

7 Varcoe C, Browne AJ, Ford-Gilboe M, et al. Reclaiming our spirits: development and pilot testing of a health promotion intervention for Indigenous women who have experienced intimate partner violence. Res Nurs Health 2017;40:237-54.

8 Browne AJ, Varcoe C, Lavoie J, et al. Enhancing health care equity with Indigenous populations: evidence-based strategies from an ethnographic study. BMC Health Serv Res 2016;16:544.

9 Bungay V. Health care among street-involved women: the perpetuation of health inequity. Qual Health Res 2013;23:1016-26.

10 Farmer P, Kim Y, Kleinman A. Introduction: A biosocial approach to global health. In: Farmer PK, ed. Reimagining global health: an introduction. CA: University of California Press, 2013: 1-14.

11 Bungay V, Johnson JL, Varcoe C, et al. Women's health and use of crack cocaine in context: structural and 'everyday' violence. Int J Drug Policy 2010;21:321-9.
12 Allen EM, Call KT, Beebe TJ, et al. Barriers to care and health care utilization among the publicly insured. Med Care 2017;55:207-14.

13 Hunter C, Fitzpatrick R, Jenkinson C, et al. Perspectives from health, social care and policy stakeholders on the value of a single selfreport outcome measure across long-term conditions: a qualitative study. BMJ Open 2015;5:e006986.

14 Robards F, Kang M, Usherwood T, et al. How marginalized young people access, engage with, and navigate health-care systems in the digital age: systematic review. J Adolesc Health 2018;62:365-81.

15 Knaak S, Mantler E, Szeto A. Mental illness-related stigma in healthcare: barriers to access and care and evidence-based solutions. Healthc Manage Forum 2017;30:111-6.

16 Clement S, Schauman O, Graham T, et al. What is the impact of mental health-related stigma on help-seeking? A systematic review of quantitative and qualitative studies. Psychol Med 2015;45:11-27.

17 Public Health Agency of Canada, and Pan-Canadian Public Health Network. Key health inequalities in Canada: a national portrait. Available: https://www.canada.ca/content/dam/phac-aspc/ documents/services/publications/science-research/key-healthinequalities-canada-national-portrait-executive-summary/hir-fullreport-eng.pdf [Accessed 28 Nov 2019].

18 World Health Organization. 10 facts on health inequities and their causes. Available: https://www.who.int/features/factfiles/health_ inequities/en/ [Accessed 28 Nov 2019].

19 Olivet J, Bassuk E, Elstad E. Outreach and engagement in homeless services: a review of the literature. The Open Health Services and Policy Journal 2010;3:53-70.

20 Arksey H, O'Malley L. Scoping studies: towards a methodological framework. Int J Soc Res Methodol 2005;8:19-32.

21 Levac D, Colquhoun H, O'Brien KK. Scoping studies: advancing the methodology. Implement Sci 2010;5:69.

22 Shamseer L, Moher D, Clarke M, et al. Preferred reporting items for systematic review and meta-analysis protocols (PRISMA-P) 2015: elaboration and explanation. BMJ 2015;349:g7647.

23 QSR International Pty Ltd. NVivo qualitative data analysis software, 2018.

24 Joanna Briggs Institute, The University of Adelaide. Critical appraisal tools. Available: https://joannabriggs.org/research/critical-appraisaltools.html [Accessed 20 Aug 2019].

25 Hsieh H-F, Shannon SE. Three approaches to qualitative content analysis. Qual Health Res 2005;15:1277-88. 